\title{
Advanced prostatic ductal carcinoma in a patient with a long survival time following a total pelvis exenteration: A case report
}

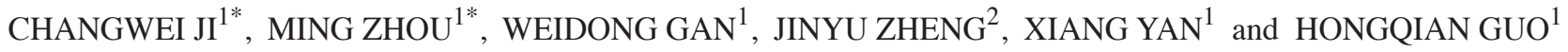 \\ Departments of ${ }^{1}$ Urology and ${ }^{2}$ Pathology, Nanjing Drum Tower Hospital, \\ The Affiliated Hospital of Nanjing University Medical School, Nanjing, Jiangsu 210008, P.R. China
}

Received February 8, 2015; Accepted December 18, 2015

DOI: $10.3892 / \mathrm{ol} .2016 .4098$

\begin{abstract}
Ductal adenocarcinoma of the prostate (DAP) is a rare variant of prostate cancer, and has poorly-identified clinical characteristics. Few cases have been previously reported in Chinese males and DAP is more commonly reported in Caucasian males over 70-years of age. In the present study, a 55-year-old Chinese male patient that demonstrated typical lower urinary tract symptoms and normal prostatic-specific antigen levels underwent a transurethral resection of the prostate. The patient was subsequently diagnosed with DAP. A total pelvis exenteration was then conducted successfully. During the 40-month follow-up, the patient remained progression-free. A review of the literature was conducted in order to assess the clinical course, diagnosis, prognosis and optimal management for DAP. The review indicated that DAP is more likely to demonstrate an aggressive clinical course and an unfavorable prognosis; therefore, once the diagnosis has been confirmed, an aggressive management strategy is recommended for the patient, even in the case of metastatic disease.
\end{abstract}

\section{Introduction}

Ductal adenocarcinoma of the prostate (DAP) was initially identified by Melicow and Patcher in 1967 (1). Initially, DAP was considered to arise from a Müllerian remnant of the prostatic utricle verumontanum (2). However, subsequent studies have clearly confirmed the prostatic origin of DAP, which is supported by a favorable response to orchiectomy, the expression of prostatic-specific antigen (PSA) and ultrastructural findings (3).

Correspondence to: Dr Weidong Gan, Department of Urology, Nanjing Drum Tower Hospital, The Affiliated Hospital of Nanjing University Medical School, 321 Zhongshan Road, Nanjing, Jiangsu 210008, P.R. China

E-mail: gwd@nju.edu.cn

${ }^{*}$ Contributed equally

Key words: prostate, ductal adenocarcinoma, radical prostatectomy, pelvis exenteration
DAP is a rare subtype of prostate cancer $(\mathrm{PCa})$ that has a poorly-understood etiology, and more commonly occurs in Caucasian males that are $>70$ years of age (4). A previous study that reviewed the histological slides of 2,600 prostatic carcinomas reported a prevalence of $0.4-0.8 \%$ for pure ductal adenocarcinoma and 5\% for mixed ductal adenocarcinoma (5). According to the latest Surveillance, Epidemiology and End Results cancer registry database, 693/731,262 patients $(0.09 \%)$ with acinar $\mathrm{PCa}$ have been identified as possessing ductal adenocarcinoma since the 1970s (6).

DAP is even more rare in the Chinese population, and few cases of DAP in Chinese men have been reported at present. The present study reports the case of a Chinese male with DAP, and reviews the relevant literature in order to summarize the clinical features, histological characteristics and therapeutic strategies for DAP.

\section{Case report}

In August 2011, a 55-year-old male presented to Nanjing Drum Tower Hospital with progressive dysuria, with urgency and frequency of urination, which had lasted for over a year. A digital rectal examination revealed a slightly swollen prostate gland, and no nodules or induration was detected. The laboratory findings included a normal urinalysis, a serum total prostate-specific antigen level of $0.27 \mathrm{ng} / \mathrm{ml}$, a tumor marker carbohydrate antigen 19-9 level of 67.47 units $/ \mathrm{ml}$ and a carcinoembryonic antigen (CEA) level of $14.40 \mathrm{ng} / \mathrm{ml}$. The prostate volume $(27.6 \mathrm{ml})$ was calculated via the transabdominal ultrasound. There was no requirement for a needle biopsy, according to the National Comprehensive Cancer Network guidelines for PCa (7). The patient was diagnosed with benign prostate hyperplasia initially and was scheduled for a transurethral resection of the prostate (TURP). During the cysto-urethroscopy, multiple gray fragile papillary lesions in the region of the verumontanum were identified protruding at the surface of the prostate. All visual lesions were resected.

Tissue samples were fixed in $10 \%$ neutral formaldehyde solution (Sigma-Aldrich Shanghai Trading Co., Ltd., Shanghai, China), dehydrated, embedded in paraffin (Leica Biosystems, Shanghai, China), and conventionally hematoxylin-eosin stained. A microscopic examination (BX41; Olympus America, Center Valley, PA, USA) of the specimen revealed that the majority of the gland was affected. The major 

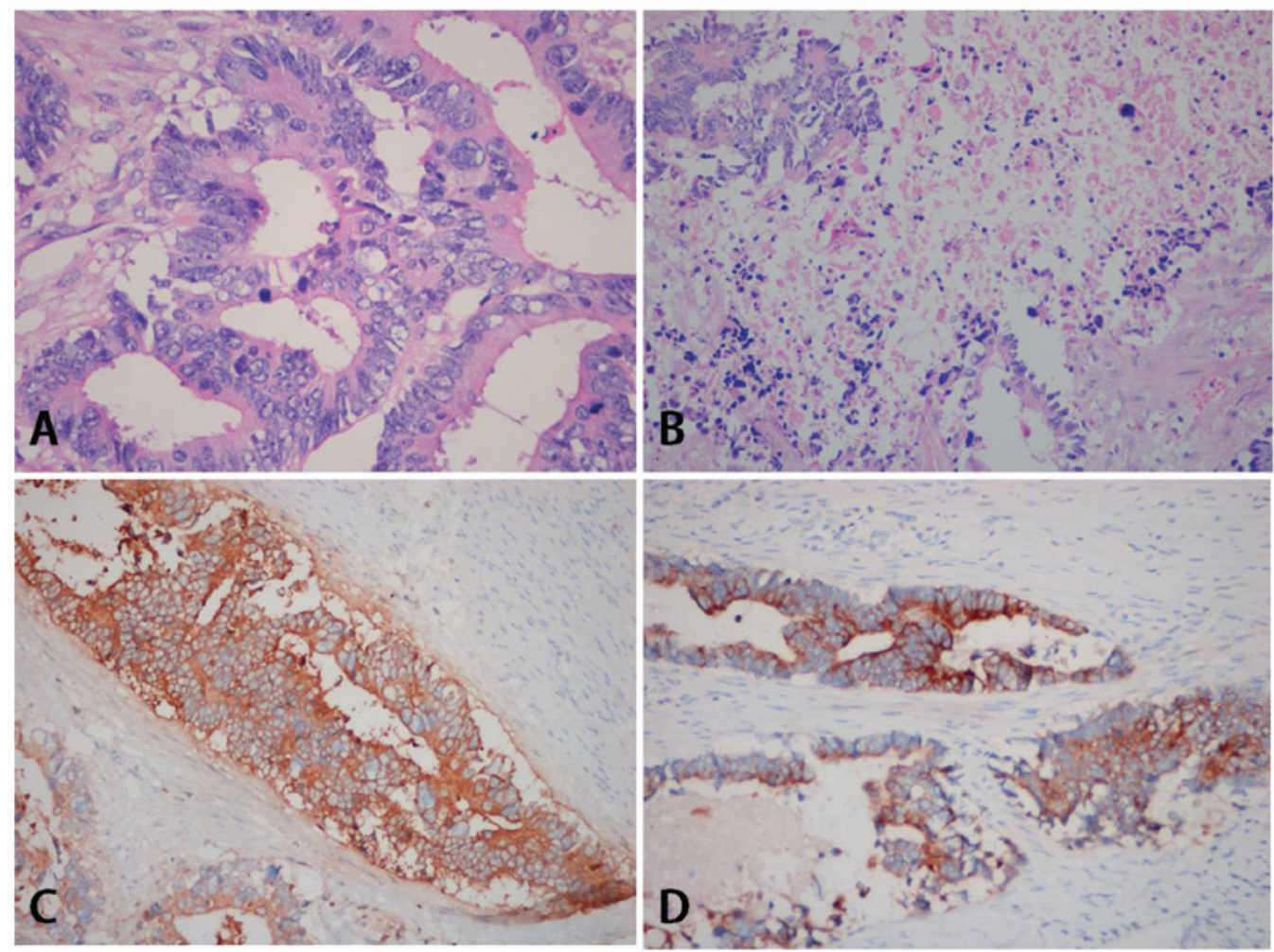

Figure 1. Typical cytological atypia of ductal adenocarcinoma of the prostate. (A) Tall pseudostratified columnar cells (HE; magnification, $\mathrm{x} 400)$. (B) Prominent necrosis and stromal fibrosis (HE; magnification, x200). (C) Expression of prostatic-specific antigen (immustaining; magnification, x400). (D) Expression of $\alpha$-methylacyl-CoA racemase (immustaining; magnification, $\mathrm{x} 400$ ). HE, hematoxylin and eosin.

components of the tumor were composed of tall pseudostratified columnar cells, with abundant eosinophilic cytoplasm, prominent necrosis and stromal fibrosis (Fig. 1). The architectural pattern was considered to be invasive glandular. The tumor cells were poorly differentiated and assigned a Gleason score of 5+4. The morphological appearance of the vascular and perineural invasion was identified. Immunohistochemical staining was performed using formalin-fixed paraffin-embedded tumor blocks. The results indicated that the expression of PSA (clone 35H9; mouse anti-human monoclonal antibody; dilution, 1:400; cat. no. PA0431; Novocastra; Leica Microsystems, Ltd., Milton Keynes, UK), $\alpha$ methylacyl CoA racemase (clone 13H4; rabbit anti-human monoclonal antibody; dilution, 1:200; cat. no. M3616; Dako, Glostrup, Denmark) and Ki67 (clone UMAB107; mouse anti-human monoclonal antibody; dilution, 1:500; cat. no. UM800033; Origene Technologies, Inc., Rockville, MD, USA), the focal expression of cytokeratin 7 (clone Ep16; rabbit anti-human monoclonal antibody; dilution, 1:250; cat. no. AC-0020; Epitomics; Abcam, Cambridge USA), cytokeratin 20 (clone Ep23; rabbit anti-human monoclonal antibody; dilution, 1:200; cat. no. AC-0026; Epitomics; Abcam) and CEA (clone COL-1; mouse anti-human monoclonal antibody; dilution, 1:150; cat. no. CM058; Biocare Medical, LLC., Concord, CA, USA), and no expression for p63 (clone UMAB4; mouse anti-human monoclonal antibody; dilution, 1:300; cat. no. UM500004; Origene Technologies, Inc.) and 34ßE12 (clone 34ßE12; mouse anti-human monoclonal antibody; dilution, 1:300; cat. no. M0630; Dako).

Subsequently, the patient underwent an ultrasonic colonoscopy. During the procedure, three polypoid lesions were identified in the sigmoid colon and descending colon regions. Additionally, a semi-circle protrusion was detected at a $10 \mathrm{~cm}$ distance from the anus. The mucous membrane of the rectum remained intact. The lesions were biopsied and removed endoscopically. The pathological findings indicated ductal adenoma associated with low-grade intraepithelial neoplasia. A bone scan and chest and abdomen computed tomography (CT) scans indicated no distant metastases. Due to the long life expectancy of the patient and the aggressive biological behavior of DAP, an exenteration of the total pelvis was performed. The surgical procedure was successful. The postsurgical specimens included the urinary bladder $(6.0 \times 5.0 \times 4.5 \mathrm{~cm})$, bilateral seminal vesicle (left, $3.0 \times 1.5 \times 1.0 \mathrm{~cm}$; right, $2.0 \times 1.0 \times 1.0 \mathrm{~cm}$ ), the entire prostate gland and the rectum $(11 \mathrm{~cm})$. The final pathological diagnosis of the mass was stage IV DAP (pT4NOM0). During the 40-month follow-up, the patient remained under close surveillance and remained progression-free with no evidence of local recurrence or distant metastasis.

Written informed consent was obtained from the patient for the publication of the present study and accompanying images.

\section{Discussion}

The diagnosis of DAP is based on cytological and immunohistochemical findings. The classic morphology is characterized by pleomorphic nuclei, abundant and usually amphophilic cytoplasm, frequent mitoses and tall pseudostratified columnar cells lined in particular architectural patterns, including papillary, cribriform, solid and invasive glandular (8). The Gleason score is the most widely accepted grading system based on 
architectural patterns. DAP is assigned a grade of at least $4+4$, due to the aggressive biological behavior and distinctive morphology (9). Regardless of variable serum PSA levels, samples that exhibit DAP histology normally express PSA and prostatic-specific acid phosphatase in immunostaining; this supports the prostatic origin of DAP and provides evidence for the differentiation of DAP tumors from other lesions. In addition, focal expression of CEA, cytokeratin 7, cytokeratin 20 and caudal type homeobox 2 have been reported in certain cases (5).

Although the clinical course of DAP remains controversial, the majority of studies indicate that the variant of PCa exhibits more aggressive biological behavior, a more advanced stage, a worse 5-year survival rate and a shorter time to progression compared with conventional acinar carcinoma $(4,6)$.

Therapies for DAP include radical prostatectomy, radiation therapy, hormone deprivation, chemotherapy and TURP. Once an initial diagnosis is confirmed using TURP or a transrectal ultrasound-guided needle biopsy, patients are advised to undergo a clinical staging evaluation, including a computed tomography scan of the abdomen and pelvis, bone scan and chest X-ray. If the diagnostic examinations rule out distant metastasis, definitive local therapies, including radical prostatectomies, pelvic lymph node dissections and external beam radiotherapy, may be offered to the patient. Notably, DAP is usually under-staged and appears to be resectable prior to surgery; however, the majority of the patients with DAP have already developed extracapsular invasion. Neoadjuvant approaches prior to surgery are recommended for patients in order to shrink the tumor volume and downstage the DAP. Patients are recommended to receive androgen deprivation following definitive therapies as DAP is prostatic in origin and androgen dependent. In one previous study, radiation therapy was recommended as a more adequate treatment option compared with radical surgery (10).
DAP is a rare entity of PCa. DAP may be challenging to detect during the early stages of disease due to the lack of distinctive clinical features, and is usually diagnosed at an advanced stage. DAP is more likely to pursue an aggressive clinical course and an unfavorable prognosis compared with other PCas. An aggressive management strategy is recommended, even for patients with metastatic disease.

\section{References}

1. Melicow MM and Pachter MR: Endometrial carcinoma of proxtatic utricle (uterus masculinus). Cancer 20: 1715-1722, 1967.

2. Carney JA and Kelalis PP: Endometrial carcinoma of the prostatic utricle. Am J Clin Pathol 60: 565-569, 1973.

3. Lee SS: Endometrioid adenocarcinoma of the prostate: A clinicopathologic and immunohistochemical study. J Surg Oncol 55: 235-238, 1994.

4. Morgan TM, Welty CJ, Vakar-Lopez F, Lin DW and Wright JL: Ductal adenocarcinoma of the prostate: Increased mortality risk and decreased serum prostate specific antigen. J Urol 184: 2303-2307, 2010

5. Epstein JI and Woodruff JM: Adenocarcinoma of the prostate with endometrioid features. A light microscopic and immunohistochemical study of ten cases. Cancer 57: 111-119, 1986.

6. Meeks JJ, Zhao LC, Cashy J and Kundu S: Incidence and outcomes of ductal carcinoma of the prostate in the USA: Analysis of data from the Surveillance, Epidemiology, and End Results program. BJU Int 109: 831-834, 2012.

7. Carroll PR, Parsons JK, Andriole G, Bahnson RR, Barocas DA, Castle EP, Catalona WJ, Dahl DM, Davis JW, Epstein JI, et al: Prostate Cancer Early Detection, Version 2.2015. J Natl Compr Canc Netw 13: 1534-1561, 2015.

8. Brinker DA, Potter SR and Epstein JI: Ductal adenocarcinoma of the prostate diagnosed on needle biopsy: Correlation with clinical and radical prostatectomy findings and progression. Am J Surg Pathol 23: 1471-1479, 1999.

9. Epstein JI: An update of the Gleason grading system. J Urol 183: 433-440, 2010

10. Orihuela E and Green JM: Ductal prostate cancer: Contemporary management and outcomes. Urol Oncol 26: 368-371, 2008. 